\title{
New generic approach for the analysis of energy conversion system models
}

\author{
Raffaele Bolliger ${ }^{\mathrm{a}}$, Helen Becker ${ }^{\mathrm{a}}$, François Maréchal ${ }^{*, a}$ \\ ${ }^{a}$ Industrial Energy Systems Laboratory (LENI) \\ Ecole Polytechnique Fédérale de Lausanne, CH-1015 Lausanne, Switzerland
}

\begin{abstract}
This paper proposes a new approach in the field of energy conversion systems analysis and synthesis. The method is based on a generic and multi-platform description syntax, that clearly separates the information concerning the physical behavior of the modeled technology (e.g. mass and energy balances and chemical reactions) from the information necessary to apply one or more system analysis methods (e.g. process integration, Life Cycle Impact Assessment, thermo-economic evaluation,... ).

The description syntax also contains other informations about the model, namely about its history, quality, scope or documentation.

The approach encourages the development of reusable models, which can be easily assembled to create large superstructures from which optimal system configurations can be extracted. By dissociating technology models from the analysis and synthesis method, the approach allows the independent development of analysis methods and the consistent data transfer between models of different scales.

The study of a fine chemical industry waste incineration system is presented to demonstrate the flexibility of the approach.
\end{abstract}

Key words: Energy conversion system analysis, computer aided process engineering, process design, process integration

\section{Introduction}

The design of energy conversion systems is based on models which describe the mass and energy balances for the different process units and their integration into the whole system. These models generate the data needed to analyze the overall system efficiency and to establish performance indicators, using for example exergy analysis, process integration with pinch analysis, life-cycle assessment or thermo-economic evaluation. The increasing complexity of the system, the highest degree of integration and the increasing number of energy conversion options together with the demand of applying different performance indicators require more systematic approaches that go beyond the use of simulation models. This paper proposes a methodology to systematically tackle the integrated system design by dissociating technology modeling from the methods for the analysis and the synthesis of integrated systems.

\section{Literature review}

In recent years, research activity in energy conversion system analysis and design evolved towards more complex and more integrated systems, often composed by combining smaller sub-systems. The domain covers multiple system scales from equipment design [11] to process design (e.g. biomass conversion processes

\footnotetext{
* Corresponding author

Email addresses: raffaele.bolliger@epfl.ch (Raffaele Bolliger), helen.becker@epfl.ch (Helen Becker), francois.marechal@epfl.ch (François Maréchal) 


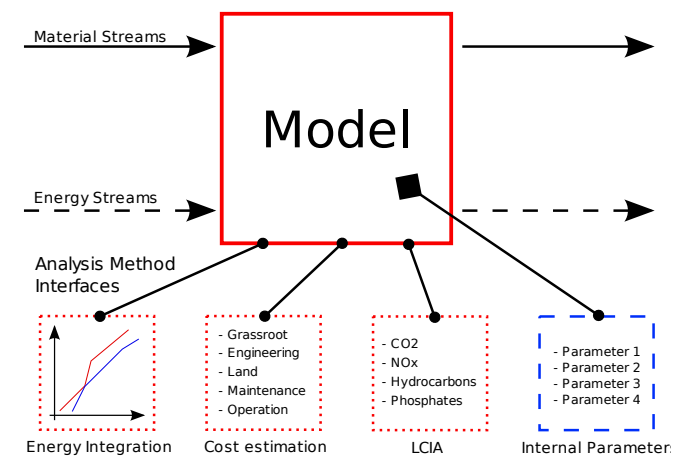

Figure 1: Schematic view of the separation of physical model from method analysis related data

[5]) to industrial processes (e.g. fine chemicals batch plants [2]) and even urban systems [10].

In order to address the problem of handling complex models, research recently focused on developing tools for exchanging informations and allow the interoperability of modeling softwares. For example, The DOME platform (distributed object-based modeling environment) [3] implements a model based co-current system design and engineering platform. It has been successfully applied to urban systems [7] allowing to interconnect versatile sub-systems models accessed from the internet using web services. The CAPE-OPEN [4] initiative on the other hand has been developed by the process engineering community to allow the interoperability of flow-sheeting tools, unit models and thermodynamic packages. Although these methods give the opportunity to construct very complex models, most of the time they are only concerned with the process flowsheet calculation problem.

The development of the system model however only solves the first part of the problem. The design of the energy conversion systems requires the application of one or more analysis and synthesis methods allowing one to deduce the performance indicators and the information about the interactions between the various technologies included in the system with a system holistic vision.

Examples of such methods are energy and process integration, life-cycle analysis, thermo-economic evaluation or exergy analysis.

The major drawback of the existing approaches is that they do not separate energy and mass balances modeling from information relative to the application of system analysis and synthesis methods. Models are built in one single block containing all the information. The reuse of the same models in different study contexts becomes therefore very difficult and often requires a partial recoding of the model.

\section{Proposed method}

We propose a new method to handle and connect models by separating the chemical and physical models of the process units from the methods used for system-scale analysis and synthesis. The method is based on a generic syntax which describes the different sub-system models and the interfaces available to model their integration in the system. A schematic representation of the concept is shown in figure 1.

The syntax provides a generic abstraction layer that describes the models independently from their modeling environment and considers their reuse in different projects. This also allows one to develop a data base of technology models that may be used to systematically develop process superstructures with interconnected technologies. 
Furthermore, the use of a generic and homogeneous syntax promotes the development of analysis and synthesis methods that are independent of the technology models, hence expanding the scope of application and allowing multiscale approaches.

A model representing a technology is the combination of a set of modeling parameters used to represent the characteristics of the technology and its environment and of a set of equations that describes the thermo-physical and chemical conversion operations of the technology. Models can be connected via their input and output streams and possibly by sharing some model parameters.

Each system analysis method applied to the model has a dedicated interface, where all the required informations are stored in a structured variable. The system analysis interfaces include the extraction and the generation of the required data for the analysis methods from the model results. For process integration application, the interface includes the definition of the hot and cold streams of the technology as well as the related energy flows (electricity, water, resources,...). For the cost estimation, the interface provides the values required to estimate the cost (e.g. the size, pressure and temperature conditions, the materials, ...). For the environmental impact assessment, the model provides information about the materials used and the size of the technology that are required to compute the direct and the indirect emissions of the technology. For the exergy analysis, not only the input, output, enthalpy and entropy are given but more detailed data are also provided such as exergy losses in the chemical reactions, pressure losses, etc... The definition of the analysis method interfaces implies therefore a detailed analysis not only of the technology itself but also of how it will interact with the other technologies in the analyzed system. This analysis is however done independently of the other technologies considered and is therefore independent of the system analyzed.

The system analysis and synthesis methods will use the system analysis interfaces to generate the performance indicators at the system level considering the possible integration of each technology in the system.

The interest of the approach is the separation of tasks: the development of a new analysis method does not require the modification of the physical model and does not affect the information handled by the other methods. However, it may require specific development when particular data are needed.

The syntax also defines other data, in relation with the inclusion of the model into a shared database. These informations concern the model classification, its documentation, the history of modifications and more generally data allowing to establish the model accuracy and quality. Table 1 defines the domains covered by the syntax.

\begin{tabular}{ll} 
Domain & Description \\
\hline Physical model & Definition of input and output flows (material and energy) crossing the model boundaries. \\
& Description of the model parameters used to control its behavior. \\
System Analysis interfaces & Definition of data required to apply system analysis and synthesis methods. \\
Classification & Information used to place the model inside searchable database. \\
Files, version & Information about model content, required software, versions. \\
Changesets, references & Information about the authors, model changes and improvements. \\
Documentation & References to model documentation and bibliographic references. \\
Validation & Information about model quality and validation status.
\end{tabular}

Table 1: Domains covered by EnergyTechnologies

The syntax defined is generic: it can be implemented in any programming language accepting structured variables; the content of the structures is suitable to describe any energy conversion technology and more generally any model which can be built with a black-box technique. 


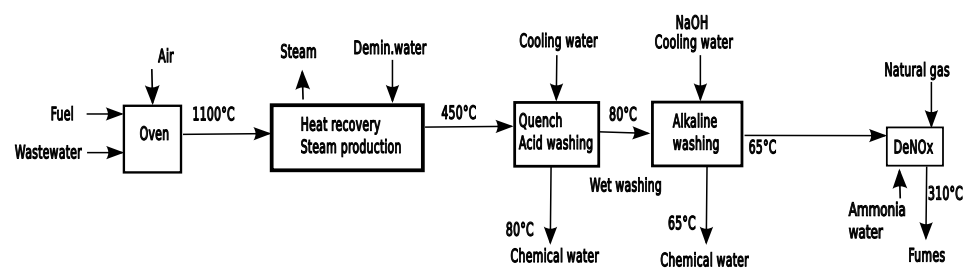

Figure 2: Flowsheet of the waste incineration plant

\section{Case study - waste incineration system}

The method has been applied to study the integration of a liquid waste incineration system composed by four units.

The first sub-system model represents the liquid waste incineration plant. The unit model includes the burner, the fumes washing devices and a deNOx (see fig. 2). The thermodynamic model is built with a commercial flow-sheeting software.

The second model represents the steam network. It includes the heat recovery steam generator, the steam turbines and the pumps. The model is formulated as a mixed integer linear programming optimization problem, where the optimal layout of the steam network in terms of electricity production is computed according to the available heat profile in the steam generator $[9,1]$.

The third sub-system model represents the cooling water system, which is used to cool down the fumes and the steam in the condenser. The fourth model represents the industrial plant where the waste incineration plant is situated. The model defines the heat requirement as the amount of steam to be produced to satisfy the heat requirement of the plant. The third and fourth models are simple representations of a cold and a hot stream, defined in the energy integration interface described in figure 1, without any model applied. When the model is available, then the unit will be substituted without having to redevelop whole model. If a new alternative method exists for treating the liquid waste (e.g. a wet oxidation process), the model will be added to the system and the system analysis will allow one to compare the two options.

An energy and process integration analysis is performed on the system in order to study the interactions between the four models. The heat recovery opportunities will be studied in order to maximize the combined steam and electricity production.

A multi-objective optimization using an inhouse advanced evolutionary algorithm [8] is performed to find the optimal layout of the steam network in order to maximize two objectives in competition: steam and electricity production. The optimization is run in an inhouse computational platform developed to apply the proposed approach[5].

This example let us show the flexibility of the approach in terms of system integration. The process integration of the utilities is visualized by the integrated composite curves (fig. 3b, 4a, 4b), while the multiobjective optimization systematically generates competing technical solutions and is an extremely valuable computer aided decision tool for the engineer.

\subsection{System analysis method: process integration}

The objective of pinch analysis and process integration is to identify the heat recovery potential between the hot and cold streams in a system [6]. All the cold and hot streams of the process have to be identified in the various units of the process and the utilities. Based on the definition of a minimum temperature difference $\left(\Delta T_{\text {min }}\right)$ the minimum energy requirement (MER) is computed. The maximum energy recovery 


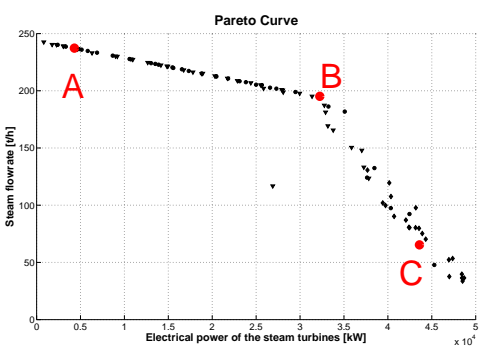

(a) Pareto curve

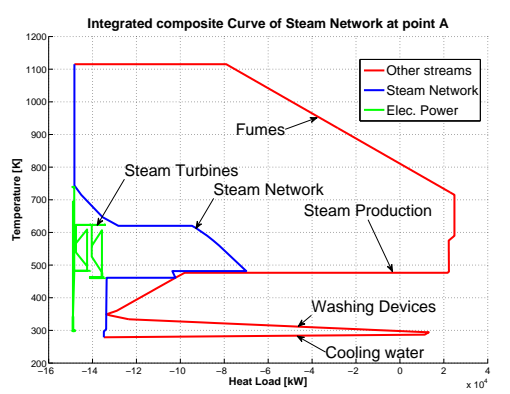

(b) Case A

Figure 3: Pareto curve of the optimization and integrated curve of case A

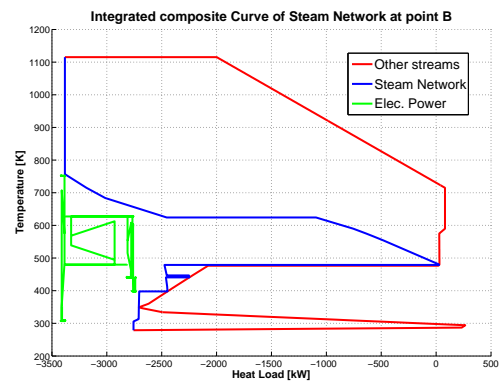

(a) Case B

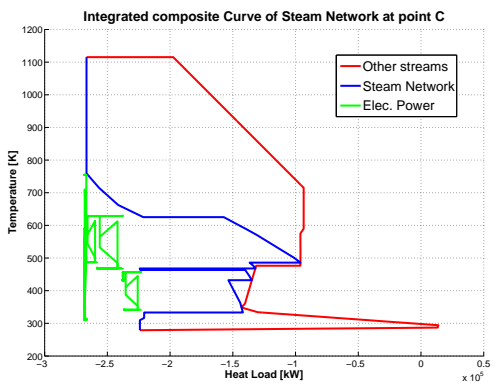

(b) Case C

Figure 4: Integrated curves of steam network for cases B and C

between the process streams is also calculated.

In the case of the proposed example, the waste incineration system and the steam requirement are defined as process units, while the steam network and the cooling water are defined as utilities whose sizes have to be optimized. The process integration model [9] computes the optimal flows in the utility system in order to satisfy the process requirement. At the same time, the mechanical power produced by the steam turbines is maximized.

The composite curves are used to analyze the solutions and to visualize the process integration. The composite curve of the hot and cold streams shows the minimum energy requirements of the process.

The grand composite curve shows if the utilities can satisfy the process requirements and displays the potential heat recovery improvements.

Integrated curves will be used to analyze the integration of a particular unit with the rest of the whole system.

\subsection{Optimization results}

The multi-objective optimization of the problem leads to a Pareto optimum set (fig. 3a) which represents the best compromise between the two objective functions. Solutions appear to span over two different zones. Table 2 displays electricity production and steam flow rates corresponding to cases A to C. Figure $3 \mathrm{~b}$ and 4 display the integrated curve of the steam network for cases A to C.

The analysis of the results shows that case B corresponds to the point where the maximum steam flow is reached in the high pressure header. The increase in electricity production going from case B to case 


\begin{tabular}{ccc} 
Case & Electricity $[\mathrm{kW}]$ & Steam $[\mathrm{t} / \mathrm{h}]$ \\
\hline A & 4644 & 236.5 \\
B & 32246 & 195.2 \\
C & 43598 & 65.3
\end{tabular}

Table 2: Comparison of solutions

$\mathrm{C}$ is performed at the expense of the steam production that quickly decreases. On the other hand, steam production is only slightly affected with the increase of electricity production at high temperature, from case A to B. Solution B is therefore the best compromise in terms of steam/electricity production ratio.

\section{Conclusions}

The proposed approach addresses the problem of the size increase in the domain of the analysis and synthesis of energy conversion systems. In order to overcome the complexity of the models, which are often developed on a per-study bases, this paper proposes a new method, which separates the modeling of a technology from the information necessary to apply system analysis methods. This is done by defining a generic and platform-independent syntax, which can be used to describe models built with commercial or inhouse packages.

The approach encourages the development of more generic and reusable models, which can be stored in a shared database and later on be reused in future studies. The proposed approach allows to uncouple the technology model development from the development of system analysis and synthesis methods whose models and tools become independent from the technology itself.

\section{References}

[1] Raffaele Bolliger, Daniel Favrat, and François Maréchal. Advanced Power Plant Design Methodology using Process Integration and Multi-Objective Thermo-Economic Optimisation. In ECOS 2005, 18th International Conference on Efficiency, Cost, Optimization, Simulation and Environmental Impact of Energy Systems, volume 2, pages 777-784, Trondheim, Norway, 2005.

[2] Raffaele Bolliger, Francesca Palazzi, and François Maréchal. Heat Exchanger Network (HEN) costs and performances estimation for multi-period operation. In 18TH European Symposium on Computer Aided Process Engineering, volume 24. Elsevier.

[3] N. Borland and D. Wallace. Environmentally conscious product design: A collaborative internet-based modeling approach. Journal of Industrial Ecology, 3(2-3):33-46, 1999.

[4] Ray Dickinson. Expanding process modelling capability through software interoperability standards: Application, extension and maintenance of cape open standards. In Bertrand Braunschweig and Xavier Joulia, editors, 18th European Symposium on Computer Aided Process Engineering, volume 25 of Computer Aided Chemical Engineering, pages 537 537. 2008.

[5] Martin Gassner and François Maréchal. Methodology for the optimal thermo-economic, multi-objective design of thermochemical fuel production from biomass. Computers and chemical engineering, 33(3):769-781, 2009.

[6] I.C. Kemp. Pinch analysis and process integration: a user guide on process integration for the efficient use of energy. Second Edition, Elsevier, Butterworth-Heinemann, UK, 2007.

[7] S. B. Kraines and D. R. Wallace. Urban sustainability technology evaluation in a distributed object-based modeling environment. Computers, Environment and Urban Systems, 27(2):143-161, 2003.

[8] G. Leyland. Multi-objective optimisation applied to industrial energy problems. PhD thesis, Ecole Polytechnique Federale de Lausanne, 2002.

[9] Francois Marechal and Boris Kalitventzeff. Targeting the optimal integration of steam networks: Mathematical tools and methodology. Computers And Chemical Engineering, 23:S133-S136, 1999.

[10] Francois Marechal, Celine Weber, and Daniel Favrat. Energy Systems Engineering, chapter Multi-Objective Design and Optimisation of Urban Energy Systems, pages 39-81. Number ISBN: 978-3-527-31694-6. Wiley, 2008.

[11] Francesca Palazzi, Nordahl Autissier, François Maréchal, and Jan Van herle. A Methodology for Thermo-Economic Modeling and Optimization of SOFC Systems. Chemical Engineering Transactions, 7:13-18, 2005. 